\title{
Deriving exact results for Ising-like models from the cluster variation method
}

\begin{abstract}
Alessandro Pelizzola ${ }^{\text {a* }}$
${ }^{a}$ Istituto Nazionale per la Fisica della Materia and Dipartimento di Fisica del Politecnico di Torino, c. Duca degli Abruzzi 24, 10129 Torino, Italy

The cluster variation method (CVM) is an approximation technique which generalizes the mean field approximation and has been widely applied in the last decades, mainly for finding accurate phase diagrams of Ising-like lattice models. Here we discuss in which cases the CVM can yield exact results, considering: (i) one-dimensional systems and strips (in which case the method reduces to the transfer matrix method), (ii) tree-like lattices and (iii) the so-called disorder points of euclidean lattice models with competitive interactions in more than one dimension.
\end{abstract}

The cluster variation method $(\mathrm{CVM})$ is a hierarchy of approximation techniques for discrete (Ising-like) classical lattice models, which has been invented by Kikuchi [1]. In its modern formulation [2] the CVM is based on the variational principle of equilibrium statistical mechanics, which says that the free energy $F$ of a model defined on the lattice $\Lambda$ is given by

$F=\min F\left[\rho_{\Lambda}\right]=\min \operatorname{Tr}\left(\rho_{\Lambda} H+\rho_{\Lambda} \ln \rho_{\Lambda}\right)$,

where $H$ is the hamiltonian of the model, $\beta=1$ for simplicity, and the density matrix $\rho_{\Lambda}$ must be properly normalized: $\operatorname{Tr}\left(\rho_{\Lambda}\right)=1$.

As a first step one usually introduces the cluster density matrices and the cluster entropies

$\rho_{\alpha}=\operatorname{Tr}_{\Lambda \backslash \alpha} \rho_{\Lambda} \quad S_{\alpha}=-\operatorname{Tr}\left(\rho_{\alpha} \ln \rho_{\alpha}\right)$,

where $\alpha$ is a cluster of $n_{\alpha}$ sites and $\operatorname{Tr}_{\Lambda \backslash \alpha}$ denotes a summation over all degrees of freedom except those belonging to the cluster $\alpha$. One then introduces the cumulant expansion of the cluster entropies

$S_{\alpha}=\sum_{\beta \subseteq \alpha} \tilde{S}_{\beta} \quad \Leftrightarrow \quad \tilde{S}_{\beta}=\sum_{\alpha \subseteq \beta}(-1)^{n_{\alpha}-n_{\beta}} S_{\alpha}$,

in terms of which the variational free energy can be rewritten as

$F\left[\rho_{\Lambda}\right]=\operatorname{Tr}\left(\rho_{\Lambda} H\right)-\sum_{\beta \subseteq \Lambda} \tilde{S}_{\beta}$.

*E-mail: alex@athena.polito.it
The above steps are all exact and the approximation defining the CVM comes in when one truncates the cumulant expansion of the entropy. The sum of the cumulants of the cluster entropies is restricted to a given set $M$ of clusters, which in most cases can be thought of as a set of maximal clusters and all their subclusters. If the model under consideration has only short range interactions and the maximal clusters are sufficiently large the hamiltonian can be decomposed into a sum of cluster contributions and the approximate variational free energy takes the form

$$
\begin{aligned}
F\left[\left\{\rho_{\alpha}, \alpha \in M\right\}\right] & \simeq \sum_{\alpha \in M}\left[\operatorname{Tr}\left(\rho_{\alpha} H_{\alpha}\right)-\tilde{S}_{\alpha}\right] \\
& =\sum_{\alpha \in M}\left[\operatorname{Tr}\left(\rho_{\alpha} H_{\alpha}\right)-a_{\alpha} S_{\alpha}\right],
\end{aligned}
$$

where the coefficients $a_{\alpha}$ can be easily obtained from the set of linear equations

$\sum_{\beta \subseteq \alpha \in M} a_{\alpha}=1, \quad \forall \beta \in M$

and the cluster density matrices must satisfy the following conditions which express normalization

$\operatorname{Tr} \rho_{\alpha}=1, \quad \forall \alpha \in M$

and compatibility

$\rho_{\alpha}=\operatorname{Tr}_{\beta \backslash \alpha} \rho_{\beta}, \quad \forall \alpha \subset \beta \in M$.

Having introduced an approximation it is worth asking whether there are special cases in 
which it turns out to be exact. The simplest example is that of a system defined on a lattice $\Lambda$ which can be regarded as the union of two clusters $\Lambda=A \cup B$, such that, denoting by $K=A \cap B$ their intersection, there is no interaction between $A^{\prime}=$ $A \backslash K$ and $B^{\prime}=B \backslash K$. In this case the hamiltonian has the general form $H=H_{A}\left(\underline{\sigma}_{A}\right)+H_{B}\left(\underline{\sigma}_{B}\right)$ and it is easy to check that the density matrix can be written as $\rho_{\Lambda}=\frac{\rho_{A} \rho_{B}}{\rho_{K}}$, which in turn implies the decomposition $S_{\Lambda}=S_{A}+S_{B}-S_{K}$ for the entropy. The CVM approximation which one obtains with the set of clusters $M=\{A, B, K\}$ leads to the same decomposition of the entropy (eq. 6 yields $a_{A}=a_{B}=1, a_{K}=-1$ ) and is therefore exact. It can be verified that this argument can be easily generalized (to several clusters sharing a common intersection) and/or iterated (to a chain of clusters $A, B, C, \ldots)$.

The above argument could be used to explain the well-known fact that CVM approximations are exact for Bethe and cactus lattices (that is, interior of Cayley and Husimi trees, respectively), which are made of links (respectively plaquettes), sharing common sites with no loops (respectively no loops larger than the elementary plaquette). However we shall leave apart tree-like lattices and turn our attention to euclidean ones, considering first one-dimensional systems (strips) and then the disorder points of higher dimensional models.

Since it is known that the Bethe-Peierls approximation (which is the lowest level CVM approximation for a model with nearest-neighbour interactions only, obtained by taking $M=$ $\{$ links, sites $\}$ ) is exact for a one-dimensional chain, one might wonder whether there is a CVM approximation which is exact for a strip of finite width. The answer is affirmative and it is interesting to note that one recovers the transfer matrix formalism. Consider a strip of width $N$ and (finite, for the moment) length $L$ and let the hamiltonian contain only translation-invariant $\mathrm{NN}$ interactions, with open boundary conditions. In the above scheme, this is an example of a chain of intersecting clusters and we can guess that a CVM approximation with the $N \times 2$ ladders as maximal clusters should be exact. Denoting by II such clusters and by I their $N \times 1$ intersections we set $M=\{\mathrm{II}, \mathrm{I}\}$ (no other subclusters enter the cumulant expansion) and in the thermodynamic limit $L \rightarrow \infty$, assuming that translational invariance is recovered, we get the variational principle

$$
\begin{aligned}
f & =\lim _{L \rightarrow \infty} \frac{F}{L} \\
& =\min \operatorname{Tr}\left(\rho_{\mathrm{II}} H_{\mathrm{II}}+\rho_{\mathrm{II}} \ln \rho_{\mathrm{II}}-\rho_{\mathrm{I}} \ln \rho_{\mathrm{I}}\right) .
\end{aligned}
$$

Denoting by $\underline{\sigma}$ and $\underline{\sigma}^{\prime}$ the two sets of degrees of freedom of the two I subclusters of a II cluster we can solve for $\rho_{\text {II }}$ and recover the transfer matrix formalism in the form

$f=-\ln \max \sum_{\underline{\sigma}, \underline{\sigma^{\prime}}} \rho_{\mathrm{I}}^{1 / 2}(\underline{\sigma}) \mathrm{e}^{-H_{\mathrm{II}}\left(\underline{\sigma}, \underline{\sigma}^{\prime}\right)} \rho_{\mathrm{I}}^{1 / 2}\left(\underline{\sigma}^{\prime}\right)$

with the normalization constraint $\sum_{\underline{\sigma}} \rho_{\mathrm{I}}(\underline{\sigma})=1$. It is interesting to note that the CVM comes with a natural fixed point algorithm [3] for finding the local miima of the free energy, which in this case reduces to the power method for finding the largest eigenvalue of the transfer matrix.

The last (and perhaps the most interesting) case we want to consider is that of disorder points. As an example we consider the square lattice Ising model with competitive interactions, with hamiltonian

$$
\begin{aligned}
H= & -K_{1} \sum_{\langle i j\rangle} \sigma_{i} \sigma_{j}-K_{2} \sum_{\langle\langle i j\rangle\rangle} \sigma_{i} \sigma_{j} \\
& -K_{4} \sum_{[i j k l]} \sigma_{i} \sigma_{j} \sigma_{k} \sigma_{l},
\end{aligned}
$$

where $K_{1}>0$ is the NN coupling, $K_{2}<0$ the next nearest neighbour coupling and $K_{4}$ the plaquette coupling. It is known [4] that in the disordered phase of this model there is an integrable subspace given by

$$
\cosh \left(2 K_{1}\right)=\frac{\mathrm{e}^{2 K_{4}} \cosh \left(4 K_{2}\right)+\mathrm{e}^{-2 K_{2}}}{\mathrm{e}^{2 K_{2}}+\mathrm{e}^{2 K_{4}}},
$$

where the free energy density is given by

$f=-\ln \left[\exp \left(-K_{4}\right)+\exp \left(K_{4}-2 K_{2}\right)\right]$.

In this subspace the $R$ matrix has an eigenvector which is a pure tensorial product and the eigenvector of the transfer matrix corresponding to the 
largest eigenvalue is also a pure tensorial product [4], and hence the density matrix and the two-site correlations are factorized. Because of this factorization (and the corresponding decomposition of the entropy) one can expect that the model can be solved exactly by the CVM and indeed this is the case. It is enough to choose $M$ $=\{$ plaquettes and their subclusters $\}$ (of course larger maximal clusters work as well) to verify eq. 13 and also to calculate the two-site correlation functions $\Gamma(x, y)=\left\langle\sigma\left(x_{0}, y_{0}\right) \sigma\left(x_{0}+x, y_{0}+y\right)\right\rangle$ :

$$
\begin{aligned}
\Gamma(x, y) & =g^{|x|+|y|}, \\
g & =\frac{\exp \left(-4 K_{2}\right)-\cosh \left(2 K_{1}\right)}{\sinh \left(2 K_{1}\right)}
\end{aligned}
$$

as well as many-site correlation functions like the plaquette correlation function $q=\left\langle\sigma_{i} \sigma_{j} \sigma_{k} \sigma_{l}\right\rangle_{\square}$

$q=\frac{\mathrm{e}^{4 K_{4}}\left(1-\mathrm{e}^{8 K_{2}}\right)+4 \mathrm{e}^{2 K_{2}}\left(\mathrm{e}^{2 K_{4}}-\mathrm{e}^{2 K_{2}}\right)}{\mathrm{e}^{4 K_{4}}\left(1-\mathrm{e}^{8 K_{2}}\right)+4 \mathrm{e}^{2 K_{2}}\left(\mathrm{e}^{2 K_{4}}+\mathrm{e}^{2 K_{2}}\right)}$.

Details, and generalizations to other models, will be reported elsewhere 河.

\section{REFERENCES}

1. R. Kikuchi, Phys. Rev. 81 (1951) 988.

2. G. An, J. Stat. Phys. 52 (1988) 727; T. Morita, J. Stat. Phys. 59 (1990) 819.

3. R. Kikuchi, J. Chem. Phys. 60 (1974) 1071; J. Chem. Phys. 65 (1976) 4545.

4. see e.g. I.G. Enting, J. Phys. C10 (1977) 1379; R.J. Baxter, J. Phys. A17 (1984) L911; H. Meyer et al, Phys. Rev. E55 (1997) 5380 and refs. therein.

5. A. Pelizzola, in preparation. 